\title{
DIGNITAS ET NOBILITAS METAPHYSICAE: OBJETO Y ARTICULACIÓN DE LA METAFÍSICA EN EL PRÓLOGO DEL COMENTARIO DE DUNS ESCOTO A LA METAFÍSICA DE ARISTÓTELES
}

\author{
Dignitas et nobilitas metaphysicae: Metaphysics's object and articulation \\ in Duns Scotus's Prologue to his commentary on Aristotle's Metaphysics \\ Héctor Hernando Salinas \\ Pontificia Universidad Javeriana (Bogotá)
}

\begin{abstract}
RESUMEN
El presente trabajo estudia dos aspectos del prólogo de Juan Duns Escoto a sus Cuestiones a la Metafísica de Aristóteles. El argumento a priori para demostrar la nobleza y dignidad de la metafísica y el tratamiento de la pregunta por el objeto científico de la metafísica y por su articulación con las otras realidades tratadas por esta ciencia. Veremos cómo el prólogo de Escoto se inscribe en una tradición interpretativa cuyos dos interlocutores principales son Tomás de Aquino y Avicena.
\end{abstract}

Palabras clave: Duns Escoto, metafísica, Aristóteles, Tomás de Aquino, Avicena.

\begin{abstract}
This paper studies two aspects of John Duns Scotus's Prologue to his Questions on the Metaphysics of Aristotle. The a priori argument to prove the nobility and dignity of metaphysics and the treatment of the question about the scientific object of metaphysics and its articulation with the other realities treated in this science. The paper also shows how Scotus's prologue follows an interpretative tradition whose principal interlocutors are Thomas Aquinas and Avicenna.
\end{abstract}

Keywords: Duns Scotus, metaphysics, Aristotle, Thomas Aquinas, Avicenna.

\section{EL PROBLEMA DE LA METAFÍSICA}

En el texto de la Metafísica leído en la clave de la determinación de la ciencia primera y de su objeto, es decir, leído a partir de la comprensión de la ciencia expuesta en los Segundos Analíticos, Aristóteles presenta al menos cuatro posibles respuestas a la pregunta por el objeto de esta ciencia que él llama filosofía primera: (a) en el libro I (A), esta se presenta como ciencia de los primeros principios y las primeras causas (2, 982a30-b5); (b) en el libro IV $(\Gamma)$ se define como ciencia del ser en tanto ser (1, 1003a20-32); (c) en el libro VI (E) se abre la posibilidad de la ciencia primera como ciencia del ser más digno de estima (1, 1026a18-32) y (d) en el libroVII (Z) se define como ciencia de la substancia (1, 1208b1-7). Por lo demás, los lectores de Le problème de l'être chez Aristote de Pierre Aubenque, saben que Aristóteles legó a la filosofía tanto la pregunta por el objeto de la metafísica como el problema del estatuto del ser, asociado precisamente al primer problema. Cuando la investigación se concentra en la identificación entre el objeto de la 
metafísica y el ser, nos enfrentamos a un problema, aparentemente sin solución, que Pierre Aubenque ha formulado en términos de una «aporía fundamental» formada por tres enunciados sostenidos por Aristóteles. La aporía consiste en que no pueden aceptarse dos de estos enunciados sin rechazar el tercero:

1) Hay una ciencia del ser en tanto ser.

2) Toda ciencia se ocupa de un género determinado.

3) El ser no es un género.

Esta doble situación dio lugar a diferentes soluciones y articulaciones de la metafísica en la Edad Media, pues unos mil doscientos años después de la edición de Andrónico de Rodas, de lo que conocemos como el texto de la Metafísica, el Occidente medieval redescubre a Aristóteles dentro del movimiento de traducciones que redefinió la historia del pensamiento científico y filosófico a partir del siglo XII. ${ }^{2}$ Hasta entonces, únicamente se conocen y se emplean las traducciones boecianas de las Categorías y del Sobre la interpretación; acompañadas de la traducción boeciana de la Isagoge de Porfirio y de los respectivos comentarios de Boecio, conjunto de textos que a partir del siglo XIII se conocen como la logica vetus. ${ }^{3}$ Sin embargo, si el Aristóteles editado por Andrónico no se identifica con la obra escrita por Aristóteles, el Aristóteles medieval tampoco se identifica plenamente con el de Andrónico. Se trata de otro Aristóteles, uno propiamente medieval.

En efecto, la apropiación de ese corpus comportó no solamente un trabajo de lectura e interpretación de las obras disponibles de Aristóteles; también lo supuso para un número importante de obras que pasaron por ser de la autoría de Aristóteles y que hoy sabemos pseudo-aristotélicas (entre ellas el célebre Liber de causis). Además, este nuevo corpus se interpretó con la ayuda de las obras del comentarismo árabe (como los también célebres comentarios de Averroes) y, para el caso de la colección de textos que conocemos bajo el nombre de Metafísica, también a la luz de la fundamental Metaphysica sive Scientia divina de Avicena, traducida a mediados del siglo XII y cuyo protagonismo no ha sido hasta ahora suficientemente subrayado. ${ }^{4}$

Con esos insumos se consolida en los siglos por venir el ejercicio del comentario escolástico a la Metafisica de Aristóteles y la pregunta por el estatuto científico, el objeto y la estructura de la metafísica. Esta pregunta va a formularse, además, desde la comprensión medieval de la ciencia derivada de la lectura de los Segundos Analíticos. Es, pues, bajo los efectos de la recepción de la herencia aristotélica, con su prescripción de una cierta teoría de la ciencia, que se construye la problemática medieval de la metafísica a partir del Aristóteles de los Segundos Analíticos. Así se inaugura la pregunta medieval por la metafísica como ciencia.

1 Aubenque, P., Le problème de l'être chez Aristote. Essai sur la problématique aristotélicienne, París, Presses Universitaires de France, 1962, p. 222.

2 Cfr. Libera, A. de, La querelle des Universaux. De Platon à la fin du Moyen Âge, París, Seuil, 1996, p. 12 y del mismo autor, La philosophie médiévale, París, Presses Universitaires de France, 1998, p. 7.

3 Cfr. Marenbon, J., «The Latin Tradition of Logic to 1100», en: D. M. Gabbay y J. Woods (eds.), Handbook of the History of Logic. Vol. 2: Mediaeval and Renaissance Logic, Amsterdam, North-Holland, 2008, p. 2.

4 Cfr. Dod, B., «Aristoteles Latinus», en N. Kretzmann, A. Kenny y J. Pinborg (eds.), The Cambridge History of Later Medieval Philosophy, Cambridge, Cambridge Univesity Press, 1982, pp. 45-79 y Trizio, M., Gutas, D. \& Burnett, Ch., «Apendix B: Medieval translations 1-3», en R. Pasnau y Ch. van Dyke (eds.), The Cambridge History of Medieval Philosophy, vol. II, Cambridge, Cambridge University Press, 2010, pp. 793-822. 


\section{EL COMENTARIO DE DUNS ESCOTO}

Sin duda, en este registro, una obra fundamental del siglo XIII es el comentario de Juan Duns Escoto a la Metafísica de Aristóteles. Solo disponemos desde 1997 de una edición crítica de este texto fundamental en la historia de la metafísica; ${ }^{5}$ y de una traducción inglesa publicada en dos volúmenes entre 1997 y 1998. ${ }^{6}$ La publicación de la edición crítica dio lugar a una lectura e interpretación renovadas de la obra. Aquí solo señalo un elemento puesto en evidencia por esta edición. Tradicionalmente, se consideraba que las Cuestiones sobre la Metafísica era un tratado temprano en la carrera de Escoto, escrito en la década de 1290, y en todo caso, con anterioridad a la redacción de la Lectura, su comentario oxoniano a los dos primeros libros de las Sententicias de Pedro Lombardo, cuya redacción se sitúa hacia 12981299. Sin embargo, las Cuestiones sobre la Metafísica contienen al menos dos estratos de redacción: uno que corresponde efectivamente a una época temprana en el pensamiento de Escoto y otra que correspondería a un desarrollo posterior de su pensamiento. Este punto era señalado ya en 1993, antes de la edición crítica, por el gran escotista americano A. B. Wolter?

La edición crítica permitió identificar sobre una base textual firme, una redacción de base o primera redacción, de un lado, y, de otro lado, una intervención posterior que puede tomar la forma de parágrafos insertados en distintos lugares de una cuestión o que se evidencia por la inserción de nuevas secciones de importante amplitud dentro de una cuestión. Junto a esta forma de intervención, que los editores llaman «adiciones» o «extras», y que en la mayoría de los casos se identifican con modificaciones introducidas por Escoto, se encuentran también cortas observaciones marginales llamadas «anotaciones interpoladas» $\mathrm{o}$ «textos interpolados». En este segundo caso, los editores consideran que no se trata, en la mayoría de los casos, de modificaciones de Escoto, sino de un tercero. ${ }^{8}$

Teniendo en cuenta esta situación textual tan particular, uno de los desafíos de toda lectura e interpretación de estos textos consiste en establecer las relaciones de convergencia y divergencia entre estas dos redacciones. En este sentido, el prólogo ${ }^{9}$ a las Cuestiones sobre la Metafísica no ofrece dificultades de fondo en cuanto a los dos estratos de redacción que comporta, pues a la aproximación al problema de la articulación de la metafísica contenida en la primera redacción, la segunda va a agregar una determinación de las causas de la metafísica, sin entrar a modificar la posición expresada en la primera redacción.

En el prólogo de Duns Escoto, encontramos además un diálogo explícito e implícito con esta tradición, y en especial, con la solución ofrecida por Tomás de Aquino en el prólogo a su propio comentario a la Metafísica. Por esta razón, consideramos que la lectura del prólogo de Escoto debe hacerse también por constraste con el de Tomás. Si desde la edición crítica sabe-

5 Juan Duns Escoto, Opera philosophica III-IV: Quaestiones super libros metaphysicorum Aristotelis, R. Andrews et al. (eds.), St. Bonaventure (NY), The Franciscan Institute, 1997.

6 John Duns Scotus, Questions on the Metaphysics of Aristotle, II Vols., translated by Girard J. Etzkorn \& Allan B. Wolter, O.F.M., St. Bonaventure (NY), Franciscan Institute Publications, 1997-1998.

7 Wolter, A. B., «Reflections on the Life and Works of Scotus», American Catholic Philosophical Quarterly, 67/1 (1993), pp. 35-36; del mismo autor, «Reflections about Scotus's Early Works», en L. Honnefelder, R. Wood \& Mechthild Dreyer (eds.), John Duns Scotus: Metaphysics and Ethics, Leyde, Brill, 1996, pp. 38 y 52 en las que el autor sostiene que ya no es posible ver en el comentario a la Metafísica una obra acabada antes de la redacción de la Lectura.

8 Introduction, § 4: «Additions, Extras, Cancelled Texts, Interpolations», en Opera philosophica III, op. cit. p. XXVIII-XXIX.

9 Fue de mucho provecho para nuestra lectura del prólogo, el artículo de Demange, D., «L'objet de la philosophie première selon Thomas d'Aquin et Jean Duns Scot», Le Philosophoire, 20 (2003), pp. 173-196. 
mos que el interlocutor privilegiado de Escoto, al menos en su obra teológica, es Enrique de Gante; en el caso de este prólogo, la relación con Tomás es directa y su evaluación necesaria. ${ }^{10}$

Señalemos desde ya una importante diferencia de énfasis entre los dos textos: el de Tomás es un comentario lineal que sigue y expone la argumentación aristotélica línea a línea buscando explicar la posición de Aristóteles, mientras el de Escoto es un comentario por cuestiones que está menos en función de entender el texto aristotélico de la Metafísica que de resolver o, al menos, plantear los problemas relativos a la metafísica misma en su constitución científica. La estructura del comentario por cuestiones permite en efecto formular preguntas más asociadas con la actualidad filosófica de Escoto y tiene menos la intención de explicar el texto que se comenta. Esto no supone un desinterés por el texto de Aristóteles, por el contrario, lo toma como punto de partida para la formulación de problemas filosóficos suscitados por su apropiación crítica. Esta distinción puede suscitar otro malentendido: que Tomás comente pero no ofrezca su propio punto de vista. Al respecto nos apoyamos en la observación de J. C. Doig, que podremos probar por nuestra cuenta en el curso de esta presentación: «it seems worthwhile to note that a Thomist conception of metaphysics is readily available to the reader of the Commentary. It is found in the Prooemium, the one part of the Commentary undeniably expressive of Aquinas' own thought». ${ }^{11}$

En cuanto a los prólogos en sí mismos, cada uno a su manera constituye una introducción condensada a la problemática de la metafísica en la Edad Media. Ambos fueron escritos como una glosa al enunciado inaugural de la Metafísica de Aristóteles: omnes homines natura scire desiderant (980a21). En cuanto a la datación, el comentario de Tomás fue probablemente compuesto entre 1270 y 1273, hacia el final de su vida. ${ }^{12}$ Mientras el de Escoto fue escrito a partir de la década de 1290 y, como ya lo señalamos, fue objeto de al menos una intervención en la que Escoto agregó nuevas consideraciones sobre las causas de la metafísica. La redacción de base del prólogo corresponde a una etapa temprana de su pensamiento, antes de que Escoto se volviera el filósofo de la univocidad del ser. Por lo demás, la intervención sobre este texto, tampoco parece alinearse sobre la tesis de la univocidad. En ese sentido, las dos redacciones que componen el prólogo parecen provenir de un periodo anterior a la tesis de la univocidad.

10 Gilson, E., Jean Duns Scot: Introduction à ses positions fondamentales, París, Vrin, 1952. p. 10, en su momento privilegió por razones que él consideraba pertienentes para una lectura filosófica de Duns Escoto, el diálogo entre Escoto y Tomás, antes que el diálogo históricamente fundado entre Escoto y Enrique de Gante: «Chercher à comprendre les positions fondamentales du Docteur Subtil n'est aucunement le situer dans son temps». En este caso preciso, el filósofo, y Gilson se presenta a sí mismo en tanto tal, «ne peut pas ne pas faire tort à la vérité de l'histoire. [...] Notre Duns Scot, dont l'interlocuteur principal est Thomas d'Aquin, n'est pas une réalité historique, mais son dialogue avec Thomas d'Aquin en est une et, de tous ses interlocuteurs, Thomas d'Aquin est philosophiquement le principal. Il l'est en soi ; il l'est donc aussi pour nous». En los últimos treinta años, este modo de leer a Escoto ha sido duramente criticado, pues opone la filosofía a su historia, y supone que la comprensión filosófica de un autor puede suspender la relación de su obra con los interlocutores efectivos que la hicieron posible. Este punto no excluye, por supuesto, que la obra discuta con otros autores que no fueron relevantes en su propia constitución. Boulnois, O., «Analogie et univocité selon Duns Scot: La double destruction», Les Études philosophiques, «L'analogie», 3-4 (1989), pp. 347-369, (aquí pp. 348-49), presenta unas importantes observaciones críticas a la posición de Gilson.

11 Doig, J. C., Aquinas on Metaphysics: A Historico-doctrinal Study of the Commentary on the Metaphysics, La Haye, Martinus Nijhoff, 1972, p. 55. Sin embargo, esta posición no es unánime entre los intérpretes del prólogo tomasiano, por ejemplo, Wippel, J. F., «Thomas Aquinas's Commentary on Aristotle's Metaphysics», en J. F. Wippel, Metaphysical Themes in Thomas Aquinas II, Washington, The Catholic University of America Press, 2007, pp. 244-245.

12 Torrell, J.-P., Saint Thomas d'Aquin: L'homme et l'œuvre. París, Les éditions du Cerf, 2012, pp. $257-258$. 
La estructura del prólogo escotista, esta comporta tres momentos. En primer lugar, una doble prueba de la dignidad y nobleza de la metafísica. La primera extraída de Aristóteles y fundada sobre el motivo del amor a los sentidos; y la segunda, extraída del comentario de Tomás y fundada en la relación entre el deseo humano y su objeto. En el segundo momento del prólogo, Escoto se pregunta cuál es el sujeto ${ }^{13}$ de esta ciencia. Y en el tercero, que corresponde a la segundo redacción, Escoto se ocupa de las causas de la metafísica.

Aquí nos vamos a concentrar en dos aspectos de este texto: en primer lugar, en su diálogo explícito con Tomás a propósito de la segunda prueba de la dignidad y nobleza de la metafísica; y en segundo lugar, en algunos desplazamientos de fondo con respecto a la articulación y objeto de la metafísica propuestas por Tomás. Daremos un paso previo que consiste en presentar el prólogo de Tomás y algunos antecedentes a la problemática del objeto de la metafísica, cuyas condiciones se derivan, en la Edad Media, principalmente de la obra de Avicena.

Notemos desde ya que Escoto implícitamente rechaza la articulación tomasiana de la metafísica, para dar lugar menos a una articulación nueva que al replanteamiento del problema de la articulación de la metafísica. En efecto, Escoto no va a responder la pregunta por la estructura de la metafísica en su prólogo, por el contrario va a reabrir la pregunta a pesar de la solución tomasiana. Para encontrar una o varias propuestas de articulación de la metafísica, hay que estudiar la primera cuestión del primer libro de su comentario..$^{14}$

\section{EL PRÓLOGO DE TOMÁS}

El sucinto prólogo tomasiano ${ }^{15}$ parte de un principio tomado de la Política de Aristóteles (1254a28-30): en toda pluralidad ordenada a un primer elemento, este último regula o rige los elementos restantes. Este principio se aplica a las relaciones entre las ciencias, que se ordenan a un único fin, la perfección del hombre, es decir, su felicidad. En consecuencia, entre las diversas ciencias debe encontrarse una ciencia primera, reguladora o regente de las otras, ciencia que Tomás llama sabiduría (sapientia).

13 El término latino subiectum es de uso común en el lenguaje técnico de la teoría de la ciencia medieval construida sobre el pensamiento de Aristóteles, particularmente los Segundos Analíticos, para referirse al dominio de objetos que delimitaba un saber científico o un campo de investigación de una ciencia determinada, un género cientîfico. En lo que sigue me referiré al subiectum scientiae con el término castellano «objeto». Para un tratamiento de este tema, ver Courtine, J.-F., Suarez et le système de la métaphysique, París, Presses Universitaires de France, 1990, pp. 10-11.

14 Juan Duns Escoto, In Met., I, q. 1 (OPh, III, 15-72). El título de esta primera cuestión dice así: «¿q. 1 el sujeto propio (proprium subiectum) de la metafísica es el ente en tanto ente, o Dios y las Inteligencias?» Y las primeras líneas de la cuestión sitúan esta problemática en el terreno de la disputa entre Avicena y Averroes, es decir, vacilando entre ontología y teología: «Sobre el objeto de esta ciencia (obiecto huius scientiae) se mostró antes que esta ciencia trata de las <realidades> trascendentes; y se mostró aue trata de las causas más elevadas. Sin embargo, hay varias opiniones sobre cuál de estos dos deba ponerse como su objeto propio (proprium eius obiectum). Por lo tanto, a este propósito se pregunta en primer lugar, si el sujeto propio de la metafísica (proprium subiectum metaphysicae) es el ente en cuanto ante (como puso Avicena) o Dios y las Inteligencias (como puso el Comentador Averroes)». In Met., I, q. 1 (OPh, III, 15).

15 Tomás de Aquino, In Duodecim Libros Metaphysicorum Aristotelis expositio, eds. M. R. Cathala y R. M. Spiazzi, Turín-Roma, Marietti, 1950, Prooemium, pp. 1-2. Cf. Para un análisis reciente Boulnois, O., «La métaphysique selon saint Thomas d'Aquin. Lecture du «Prologue » de son commentaire d'Aristote», en T.-D. Humbrecht (dir.), Saint Thomas d'Aquin, París, Les Éditions du Cerf, 2010, pp. 37-88. Y para una visión de conjunto de la metafísica tomasiana, en la que el prólogo del que nos ocupamos juega el rol de hilo conductor, Courtine, J.-F., Suarez et le système de la métaphysique, op. cit., pp. 31-74. 
Retengamos el criterio que la define: «esta ciencia debe ser, por naturaleza, reguladora de las otras, pues es <la ciencia> intelectual en grado sumo (maxime intellectualis). Y es ella la que se ocupa de las realidades inteligibles en grado sumo (maxime intelligibilia)»(p. 1). Nunca será suficiente el énfasis con que se subraye esta relación entre el sujeto de la metafísica y su inteligibilidad en grado sumo, pues es por este grado de inteligibilidad que un objeto se determina como el sujeto de la metafísica. ${ }^{16}$ Tomás busca entonces identificar el sujeto de esta ciencia, identificando lo que él llama las realidades maxime intelligibilia. Esto se ve con claridad al considerar los tres enfoques, utilizados por Tomás, que dan lugar a la identificación de tres «objetos» de los que se ocuparía la sabiduría.

(a) El primer enfoque, que parte del orden del inteligir (ordo intelligendi), se ocupa de la certitud científica y de su fuente. El intelecto alcanza la certeza a partir de las cosas que parecen más inteligibles (intelligibilia magis) y la fuente de la certeza se adquire con el conocimiento de las causas. Y puesto que se trata de alcanzar la certeza en grado sumo, la ciencia que se ocupa de las primeras causas «parece ser reguladora en grado sumo de las otras» (videtur esse maxime aliarum regulatrix) (p. 1).

(b) El segundo enfoque descansa en la comparación entre intelecto y los sentidos (ex comparatione intellectus ad sensum). Siendo universal el objeto del intelecto, «esta ciencia es intelectual en grado sumo, en tanto se ocupa de los principios universales en grado sumo» (p. 1). El objeto que se alcanza por este enfoque es el ente (ens) y todo aquello que lo sigue (ea quae consequuntur ens), por ejemplo, lo uno y lo múltiple, la potencia y el acto (y las otras propiedades trascendentales), y puesto que ellos constituyen el grado máximo de universalidad, constituyen las realidades inteligibles en grado sumo (maxime intelligibilia).

(c) El tercer enfoque se funda en el conocimiento del intelecto (ex ipsa cognitione intellectus), cuyo rasgo diferencial reposa sobre su grado de inmaterialidad o separación de la materia. Según Tomás «puesto que toda realidad posee una potencia intelectiva en la medida en que está exenta de materia, tienen que ser inteligibles en grado sumo (maxime intelligibilia) las [realidades] que están separadas de la materia en grado sumo» (p. 1). Estas realidades serán rápidamente identificadas con Dios y las Inteligencias, las entidades dotadas de inteligencia y desprovistas de cuerpo o de materia. De allí se puede concluir que las realidades inteligibles en grado sumo, las maxime intelligibilia, son las realidades separadas en grado sumo, las maxime separata.

A pesar de la diversidad de estos tres objetos, Tomás afirma la unidad de esta ciencia. ¿Cómo es posible la convergencia de estos tres objetos en una sola ciencia? Tomás comienza por establecer una primera equivalencia entre el objeto obtenido por el criterio del grado sumo de inmaterialidad (Dios y las Inteligencias) y el obtenido por el criterio del grado sumo de certeza (los primeros principios y las primeras causas). En seguida, hará de estos dos objetos, en su convergencia, la causa del objeto obtenido por el criterio de la universalidad en grado

16 Courtine, J.-F., Suarez et le système de la métaphysique, op. cit., p. 34, subraya la relevancia de la referencia a la Política de Aristóteles, pues con ella, Tomás establece el vínculo entre, de un lado, las relaciones amoesclavo, alma-cuerpo, regente-gobernado, y del otro, la relación del intelecto a lo inteligible, en el sentido de que la metafísica está orientada por una problemática directiva o reguladora, gobernadora, del intelecto. Doig, J. C., Aquinas on Metaphysics, op.cit., p. 55, se sorprende por el caracter marcadamente intelectualista del prólogo tomasiano: «The Prooemium is a strange piece of philosophical writing. Due to his unusual emphasis on the intellect...». Y, en otro sentido, Mansion, S., « L'intelligibilité métaphysique d'après le Prooemium du commentaire de saint Thomas à la Métaphysique d'Aristote », Rivista di Filosofia Neo-scolastica, LXX, 1-2 (1978), pp. 49-62, define la perspectiva del prólogo como «inteligibilidad metafísica», concebida como criterio de determinación de los objetos de esta ciencia. 
sumo (el ente): «Pues, las substancias separadas que hemos mencionado son universales y son las primeras causas del ser» (primae causae essendi) (p. 1).

En esta línea se sintetiza una articulación posible de las distintas realidades tratadas por la metafísica. En tanto que causas y principios primeros, Dios y las inteligencias son causas primeras y universales del ente, que será entonces su efecto primero. ${ }^{17}$ Corresponde pues a la misma ciencia ocuparse de un género y de las causas de ese género, como en el caso de la física que se ocupa del cuerpo natural y de sus principios. «Es por ello, que corresponde a la misma ciencia considerar las substancias separadas y el ente común, pues éste es el género del cual las mencionadas causas comunes y universales son las causas». Por este doble movimiento, Tomás vincula a Dios y a las Inteligencias al objeto de esta ciencia en tanto que causas del objeto y por el mismo gesto los distingue de él. Sin mencionarla, Tomás resuelve la aporía fundamental enunciada en el libro E de la Metafísica (1026a25-32), donde la determinación del objeto de la metafísica vacila entre lo más universal y el género que corresponde a las realidades más dignas de estima o, entre el ente generalísimo y Dios. Con esta solución ambos entran en el dominio metafísico, de un lado lo general, el ente, como objeto de esta ciencia, y del otro, las realidades más dignas de estima, Dios y las Inteligencias, como causa del objeto.

Esta unidad encontrada, y que gira alrededor del ens commune como objeto de la sabiduría, no impide a Tomás establecer la lista de los nombres posibles para esta ciencia, en función de cada uno de los enfoques considerados.

En conformidad, pues, a los tres [enfoques] mencionados, según los cuales se considera la perfección de esta ciencia, ella recibe tres nombres. Se la llama, en efecto, ciencia divina o teología, en tanto considera las substancias de las que hemos hablado; metafísica, en tanto considera al ente y lo que sigue al ente (pues estas realidades transfísicas se descubren por la vía del análisis (via resolutionis)), ${ }^{18}$ como las realidades más comunes después de las menos comunes); y se la llama filosofía primera, en tanto considera las primeras causas de las cosas. (In XII Met., Prooemium, éd. Cathala, p. 2). ${ }^{19}$

17 Cfr. Los análisis de Courtine, J.-F., Suárez et le système de la métaphysique, op. cit., pp. 39-41, quien llama la atención sobre este rasgo central de la empresa metafísica tomasiana. En ese sentido, Wood, R., «The Subject of the Aristotelian Science of Metaphysics», en R. Pasnau y Ch. van Dyke (eds.), The Cambridge History of Medieval Philosophy, vol. II, Cambridge, Cambridge University Press, 2010, p. 612, distingue cuatro tipos de aproximación a la metafísica en la Edad Media: (a) en un caso, la metafísica no tendría un objeto unificado, sino muchos sujetos. Esta sería la posición de Ockham; (b) en otra solución, Dios no sería ni objeto ni parte del objeto de la metafísica, sino causa del objeto, tal como lo acabamos de constatar en Tomás de Aquino; (c) según otro enfoque, Dios sería una parte del objeto de la metafísica, esta sería una posición atribuible a Duns Escoto en su obra teológica, por ejemplo en Ordinatio I, d. 3 qq. 1-3 (Vat. III, 1-123); y (d) finalmente, otra solución defiende la substancia como objeto de la metafísica. R. Wood presenta como uno de los representantes de esta solución, relativamente rara, a Richard Rufus de Corwall (pp. 612-614). Duns Escoto también sostiene esta tesis durante su periodo de juventud. Esta se encuentra en la primera cuestion del primer libro de su comentario a la Metafísica (In Met., I, q. 1 nn. 91-109, OPh, III, 46-53). De otro lado, Wood aclara que ella introduce esta cuarta solución a la clasificación en tres soluciones propuestas por Zimmermann, A., Ontologie oder Metaphysik? Die Diskussion über den Gegenstand der Metaphysik im 13. Und 14. Jahrhundert. Texte und Untersuchungen, Leyde-Cologne, Brill, 1965, pp. 220-22; 294-329 et 389-398.

18 Cf. sobre la via resolutionis Doig, J. C., op. cit., pp. 64-77 y Aertsen, J. A., «Method and Metaphysics: The via resolutionis in Thomas Aquinas», The New Scholasticism, 63/4 (1989), pp. 405-418.

19 Tomás de Aquino, In XII Met., Prooemium (ed. Cathala, p. 2): «Secundum igitur tria praedicta, ex quibus perfectio huius scientiae attenditur, sortitur tria nomina. Dicitur enim scientia divina sive theologia, inquantum praedictas substantias considerat. Metaphysica, inquantum considerat ens et ea quae consequuntur ipsum. Haec enim transphysica inveniuntur in via resolutionis, sicut magis communia post minus communia. Dicitur autem prima philosophia, inquantum primas rerum causas considerat. Sic igitur patet quid sit subiectum huius scientiae, et qualiter se habeat ad alias scientias, et quo nomine nominetur». 
Como vemos, la diversidad de enfoques y de apelaciones que recibe esta ciencia no pone en peligro su unidad, pues esta se funda en su objeto, el ente, del que los primeros principios y las primeras causas, es decir, Dios y las Inteligencias, son la causa.

\section{EL TRANSFONDO AVICENIANO}

Con respecto a esta triple consideración de la sabiduría, O. Boulnois llama la atención sobre el transfondo aviceniano del que depende. ${ }^{20}$ En efecto, Tomás reestructuró algunos motivos ya presentes en las primeras páginas de la Metafísica de Avicena, de la cual, Domingo Gundisalino, ${ }^{21}$ en su De divisione philosophiae, ofrece una síntesis que pudo servir de intermediario entre Avicena y Tomás. ${ }^{22}$ Estos mismos motivos se encuentran como transfondo del texto y de la problemática metafísica en el prólogo del comentario escotista.

Avicena sostiene en las primeras líneas de su Metafísica que después de haber tratado de las «intenciones» de las ciencias lógicas, físicas y matemáticas, es el momento de abordar el conocimiento de las intenciones que se ocupan de las realidades de la sabiduría (intentionum sapientialium). ${ }^{23}$ Es claro que para Avicena, así como para Tomás, la apuesta consiste en determinar el contenido de la sabiduría, su intentio, en tanto que dominio independiente de las otras ciencias teóricas, incluida la lógica.

Avicena recuerda la distinción entre las ciencias filosóficas especulativas (speculativae) y las ciencias filosóficas prácticas. En seguida, distingue las ciencias especulativas en ciencias físicas (naturales), matemáticas (doctrinales) y divinas (divinas). Allí, por primera vez, Avicena reclama para esta ciencia el nombre de «divina». Además, si para la física y la matemática, Avicena presenta (a) sus objetos (el dominio que circunscribe cada una de estas ciencias) y (b) aquello que se investiga en ellas, lo que busca demostrarse de su objeto, para el caso de la ciencia divina, Avicena solo presenta aquello de lo que se ocupa la investigación, pero guarda silencio sobre su objeto. Así, el objeto de la física son los cuerpos en tanto móviles y en reposo; y aquello que se investiga en la física son los accidentes de los cuerpos en tanto móviles y en reposo. Por su parte, el objeto de la matemática es o «la cantidad pura» o aquello

20 Boulnois, O., «La métaphysique selon saint Thomas d'Aquin. Lecture du «Prologue» de son commentaire d'Aristote», op. cit., p. 52 ss: «il est clair que le prologue de Thomas est une synthèse de la Métaphysique I, 1 d'Avicenne, qui suppose une connaissance directe et non simplement via Gundissalinus». La cursiva es mía. Sobre la relación entre Tomás y Avicena (e incluso Escoto) a propósito de la problemática metafísica, el artículo de Wippel, J. F., «The Latin Avicenna as a Source for Thomas Aquinas's Metaphysics», en J. F. Wippel, Metaphysical Themes in Thomas Aquinas II, op. cit., pp. 33-43.

21 Por ejemplo, Dominicus Gundissalinus, De divisione philosophiae (éd. Baur, 35-36): «Quia enim sapiencia describitur hiis tribus proprietatibus, que sunt: "sapiencia est nobilior sciencia, qua scitur id, quod est nobilius scitum"; item: "sapiencia est cognicio, que est certior et nobilior"; item: "sapiencia est sciencia primarum causarum tocius esse"; - hee autem tres proprietates sapiencie conueniunt huic sciencie: ergo ipsa est sapiencia, que est nobilior sciencia, qua comprehenditur nobilius scitum, nobilior sciencia est, quia est certitudo ueritatis, et nobilius scitus, quia est deus et cause que sunt post eum, et eciam cognicio causarum ultimarum omnis esse et cognicio dei».

22 Sobre la relación de dependencia de Tomás con respecto al De divisione philosophiae de Gundissalinus, O. Boulnois cambió recientemente de opínión en el capítulo 3, «Structure et diversité des métaphysiques: trois modèles» de su trabajo Métaphysiques rebelles: Genèse et structures d'une science au Moyen Âge, París, Presses Universitaires de France, 2013, pp. 132-133; y infra p. 140: «Dans le prologue de son commentaire de la Métaphysique, Thomas paraphrase les analyses de Gundissalinus, lui-même inspiré par Avicenne». Las cursivas son mías.

23 Avicenna, Philosophia prima sive scientia divina, I, 1 (AvL, I, 1, 4-6): «Postquam, auxilio Dei, explevimus tractatum de intentionibus scientiarum logicarum et naturalium et doctrinalium, convenientius est accedere ad cognitionem intentionem sapientialium». Las cursivas son mías. 
que posee una cantidad; y aquello que se investiga en matemáticas son los accidentes de la cantidad, cuya definición no tiene en cuenta ni la materia ni el movimiento. ${ }^{24}$

En cuanto a la ciencia divina, Avicena presenta al menos tres características de lo que ella investiga:

(a) En primer lugar, la ciencia divina «solo investiga las cosas separadas de la materia según el ser y la definición». ${ }^{25}$ Sin embargo, «ya has escuchado también que la ciencia divina es aquella en la cual se investigan las causas primeras del ser físico y del ser matemático y lo que depende de ellas, así como la causa de las causas y el principio de los principios que es Dios altísimo». ${ }^{26}$

Esta primera caracterización de la ciencia divina, al considerar lo que se investiga en ella, está construida a partir de la idea de separación de la materia, lo que permitirá una forma de jerarquización de las ciencias teóricas, en la que la ciencia divina (ciencia sobre Dios, en tanto Dios es causa de las causas y principio de los principios) será también causa de las otras ciencias teóricas. Esta es la dimensión «teológica» de la ciencia divina.

(b) Sin embargo, también es posible caracterizar esta ciencia como «la filosofía certísima y filosofía primera y que ella misma permite alcanzar la verificación de los principios de las otras ciencias, y que ella misma es sabiduría certísima». ${ }^{27}$ Con este caracterización, Avicena introduce los temas vinculados a la sabiduría como filosofía primera y como saber con el grado máximo de certeza. Avicena no tardará en introducir una definición de la sabiduría a partir de las ideas de «la ciencia superior» y del objeto cognoscible superior (excellentius scitum), y en esto anticipa las realidades maxime intelligibilia del prólogo de Tomás (quien leyó a Avicena) y, como fuente primera, recuerda las realidades maxime scibilia del mismísimo texto aristotélico, ${ }^{28}$ que pronto encontraremos en el prólogo de Escoto.

A menudo has escuchado también, que la sabiduría es la ciencia superior (scientia excellentior) al conocer el objeto cognoscible supremo (excellentius scitum), y además, que la sabiduría es el conocimiento de mayor certeza (certior) y el más exacto (convenientior), y además que ella misma es la ciencia de las primeras causas de todo. ${ }^{29}$

24 Avicenna, Philosophia prima sive scientia divina, I, 1 (AvL, I, 2, 20-28): «Et diximus quod speculativae comprehenduntur in tres partes, in naturales scilicet et doctrinales et divinas; et quod suum subiectum naturalium est corpora, secundum quod moventur et quiescunt, et quod de eis inquiritur est accidentia quae accidunt eis proprie secundum hunc modum; et quod suum subiectum doctrinalium est vel quod est quantitas pure, vel quod est habens quantitatem, et dispositiones eius quae inquiruntur in eis sunt ea quae accidunt quantitati ex hoc quod est quantitas, in definitione quarum non invenitur species materiae nec virtus motus».

25 Avicenna, Philosophia prima sive scientia divina, I, 1 (AvL, I, 2, 28-30): «et quod divinae scientiae non inquirunt nisi res separatas a materia secundum existentiam et definitionem».

26 Avicenna, Philosophia prima sive scientia divina, I, 1 (AvL, I, 2,28-3,34): «Iam etiam audisti quod scientia divina est in qua quaerunt de primis causis naturalis esse et doctrinalis esse et de eo quod pendent ex his, et de causa causarum et de principio principiorum, quod est Deus excelsus».

27 Avicenna, Philosophia prima sive scientia divina, I, 1 (AvL, I, 3,44-46): «Et etiam iam audisti quod haec est philosophia certissima et philosophia prima, et quod ipsa facit acquirere verificationem principiorum ceterarum scientiarum, et quod ipsa est sapientia certissime».

28 Aristóteles, Metaphysica, I (A), 2, 982a29-b2 (AL, XXV², 10): «Est et doctrinalis que causarum est speculatrix magis (hii namque docent qui causas de singulis dicunt), et noscere et scire earum causa maxime est maxime scibilis scientie (nam qui scire propter idem ipsum desiderat ipsam maxime scientiam maxime desiderabit, talis autem est ipsa maxime rei scibilis); sed sunt maxime scibilia que prima et cause». Las cursivas son mías.

29 Avicenna, Philosophia prima sive scientia divina, I, 1 (AvL, I, 3,46-50): «Iam etiam audisti saepe quod sapientia est excellentior scientia ad sciendum id quod est excellentius scitum, et iterum quod sapientia est cognitio quae est certior, et iterum quod ipsa est scientia primarum causarum totius». 
En este pasaje, se enumeran tres rasgos de la sabiduría: (a) ciencia del objeto cognoscible supremo; (b) ciencia certísima y exactísima y (c) ciencia de las primeras causas. Allí, una vez más, encontramos la fuente de la referencia a las realidades maxime scibilia de Tomás, el criterio de la certitud máxima y las primeras causas como objeto de esta ciencia. Sin embargo, incluso si percibimos la cercanía, la articulación aviceniana no coincide con la de Tomás, quien mantiene los tres rasgos de la sabiduría, mientras Avicena va a mostrar que estas tres propiedades pertenecen a un único cuerpo de doctrina (magisterium), de tal modo que evita la dispersión de los objetos y garantiza por ese mismo movimiento la unidad de la ciencia. ${ }^{30}$

Un poco más adelante, después que determina el ser como objeto de la metafísica, Avicena vuelve sobre los nombres de esta ciencia y sus rasgos principales:

Y esta es $<$ A $>$ la filosofía primera, pues ella misma es la ciencia de la causa suprema del ser, y esta es la causa primera, pero la primera causa de la totalidad (universitatis) es el ser y la unidad; y ella es también $<\mathrm{B}>$ sabiduría, pues es la ciencia más noble (nobilior scientia) por la cual es aprehendido el objeto cognoscible más noble (nobilius scitum): en efecto, ella es la ciencia más noble, pues ella es la certeza de la verdad, y a cerca del objeto cognoscible más noble, pues él es Dios y las causas que lo siguen; así mismo, es el conocimiento de las causas últimas de todo ser (omnis esse), y conocimiento de Dios, y por esta razón $<\mathrm{C}>$ la ciencia divina es definida en tanto ella es la ciencia de las cosas separadas de la materia por el límite y la definición, ${ }^{31}$ pues no hay nada del ser en tanto ser, ni de sus principios y sus accidentes, en tanto son, como ya es manifiesto, que no sea anterior a la materia y cuyo ser no dependa de ella. ${ }^{32}$

Así, Avicena presenta la filosofía primera bajo dos aspectos: en tanto ciencia de la causa primera y en tanto cienciea de la totalidad del ser. Vemos claramente que la filosofía primera en Avicena, presenta unificado lo que está separado en Tomás, bajo los nombres de filosofía primera (que se ocupa de las primeras causas en tanto fuentes de la máxima certeza) y metafísica (que se ocupa del ser en tanto principio de máxima universalidad).

Y al mismo tiempo, la sabiduría, la ciencia más noble, también está presente bajo dos aspectos: en tanto certeza de la verdad (certitudo veritatis) y en tanto ella se ocupa del objeto

30 Avicenna, Philosophia prima sive scientia divina, I, 1 (AvL, I, 3,50-4,56): «Et tamen non intellexisti qui esset haec philosophia vel haec sapientia, nec si haec tres definitiones vel proprietates sint unius artis vel diversarum quarum unaquaeque dicatur sapientia. Nunc autem nos manifestabimus quod haec scientia in cuius via sumus est philosophia prima et quod ipsa est sapientia absolute, et quod tres proprietates per quas describitur sapientia, sunt proprietates unius magisterii, et quod ipsa est ipsum magisterium».

31 En este punto seguimos a Boulnois, O., «La métaphysique selon saint Thomas d'Aquin», op. cit. p. 53, n. 3, quien sigue la lectio de los manuscritos PF (a materia termino et definitione) en lugar de la lectio, ininteligible, propuesta por el texto crítico (a materia definitione et definitionibus). O. Boulnois reenvía también a la traducción de Olga Lizzini (Avicenna, Metafisica : La scienza delle Cose Divine, trad. italiana del árabe, con texto latino; introducción, notas y aparato de O. Lizzini; prefacio y edición de P. Porro, nueva edición, Milan, Bompiani, 2006), donde se lee: «le compete la definizione di scienza divina, che è scienza delle cose che sono separate dalla materia nella definizione e nell'esistenza». p. 41. Las cursivas son mías.

32 Avicenna, Philosophia prima sive scientia divina, I, 2 (AvL, I, 15,89-16,99): «Et haec est philosophia prima, quia ipsa est scientia de prima causa esse, et haec est prima causa, sed prima causa universitatis est esse et unitas; et est etiam sapientia quae est nobilior scientia qua apprehenditur nobilius scitum: nobilior vero scientia, quia est certitudo veritatis, et nobilius scitum, quia est Deus, et causae quae sunt post eum; et etiam cognitio causarum ultimarum omnis esse, et cognitio Dei, et propterea definitur scientia divina sic quod est scientia de rebus separatis a materia definitione et definitionibus, quia ens, inquantum est ens, et principia eius et accidentia eius, inquantum sunt, sicut iam patuit, nullum eorum est nisi praecedens materiam nec pendet esse eius ex esse illius». Para las últimas líneas de este pasaje nos apoyamos en la traducción de Boulnois O., en, «La métaphysique selon saint Thomas d'Aquin», Ibid.pp. 53-54. 
cognoscible más noble, que no es otro que Dios y las causas que lo siguen. Por último, la ciencia divina, definida por el criterio de separación de la materia, se ocupa, paradódijamente, del ser en tanto ser y no de Dios. Como se puede observar, Avicena no utiliza el nombre metafísica, y sin embargo, los elementos en juego en la articulación de la metafísica están allí presentes: Dios, el ser y las primeras causas.

\section{METAFÍSICA Y FIN ÚLTIMO DEL HOMBRE}

De las dos pruebas presentadas por Tomás para demostrar la dignidad y nobleza de la metafísica, nos vamos a concentrar en la segunda, la prueba a priori, en la queTomás presenta tres argumentos cuyo acento recae sobre el objeto del deseo natural.

La estructura de los tres argumentos es muy precisa: cada uno descansa sobre un principio a propósito del deseo natural (ésta es la premisa mayor del argumento). Este principio es aplicado al caso del hombre (constituyendo la menor), de donde se puede demonstrar que al hombre corresponde el deseo natural del objeto enunciado en la premisa mayor.

(a) El primer argumento se funda en el deseo natural de perfección. En el caso del hombre, su lugar de emergencia es el intelecto, pues, el intelecto es aquello por lo quel «el hombre es lo que es», y, considerado en sí mismo, es «en potencia todas las cosas» y únicamente se actualiza por la ciencia. Siendo pues el elemento definicional del hombre, su actualización y, en consecuencia su perfección, sólo se alcanzan por la ciencia, específicamente por el acto de inteligir. Por lo tanto, todo hombre desea la ciencia (desiderat scientiam). ${ }^{33}$

(b) El segundo argumento reposa en el deseo natural del hombre de actualizar su operación propia: el acto de inteligir. «Es por ello, que el deseo natural del hombre se inclina a inteligir (ad intelligendum), y en consecuencia a saber (ad sciendum)». ${ }^{34}$

(c) El tercer argumento se apoya en el deseo natural del hombre de unirse a su principio. Y para el hombre, las substancias separadas son su principio. Ahora bien, el hombre sólo puede unirse a las substancias separadas por la mediación del intelecto. Esta es la felicidad del hombre «y es por ello que el hombre desea naturalmente la ciencia (desiderat scientiam)». ${ }^{35}$

Notemos que los tres argumentos convergen en el acto del intelecto, que constituye al mismo tiempo la perfección del hombre, su operación propia y el medio para unirse a su principio. En la arquitectura del prólogo, el tercer argumento funciona como clave de bóveda pues su conclusión apuntala el comienzo del prólogo, al hacer de la unión del hombre a su principio

33 Tomás de Aquino, In XII Met., I, Lec. 1 n. 2 (ed. Cathala, p. 6): «Primo quidem, quia unaquaeque res naturaliter appetit perfectionem sui. Unde et materia dicitur appetere formam, sicut imperfectum appetit suam perfectionem. Cum igitur intellectus, a quo homo est id quod est, in se consideratus sit in potentia omnia, nec in actum eorum reducatur nisi per scientiam, quia nihil est eorum quae sunt, ante intelligere, ut dicitur in tertio de anima: sic naturaliter unusquisque desiderat scientiam sicut materia formam».

34 Tomás de Aquino, In XII Met., I, Lec. 1 n. 3 (ed. Cathala, p. 6): «Secundo, quia quaelibet res naturalem inclinationem habet ad suam propriam operationem: sicut calidum ad calefaciendum, et grave ut deorsum moveatur. Propria autem operatio hominis inquantum homo, est intelligere. Per hoc enim ab omnibus aliis differt. Unde naturaliter desiderium hominis inclinatur ad intelligendum, et per consequens ad sciendum».

35 Tomás de Aquino, In XII Met., I, Lec. 1 n. 3 (ed. Cathala, p. 6): «Tertio, quia unicuique rei desiderabile est, ut suo principio coniungatur; in hoc enim uniuscuiusque perfectio consistit. Unde et motus circularis est perfectissimus, ut probatur octavo physicorum, quia finem coniungit principio. Substantiis autem separatis, quae sunt principia intellectus humani, et ad quae intellectus humanus se habet ut imperfectum ad perfectum, non coniungitur homo nisi per intellectum: unde et in hoc ultima hominis felicitas consistit. Et ideo naturaliter homo desiderat scientiam». 
la actualización de su perfección y la posesión de la felicidad (felicitas). El acto propio del hombre y su perfección se sitúan y se comprenden en función del fin del hombre: la unión o el retorno al principio. Aparentemente, por este último argumento se puede establecer el vínculo entre las realidades inteligibles en grado sumo (maxime intelligibilia), ahora identificadas con las substancias separadas, y la búsqueda de la sabiduría.

Tal como Escoto expone y analiza estas pruebas en su propio prólogo, ellas constituyen una progresión hacia una única conclusión y por lo tanto forman un único argumento. El primer argumento sostiene el deseo natural de saber en tanto acto primero del alma (In Met. I, Prol.n. 5 OPh, III, 4-5); el segundo sostiene el deseo natural de inteligir, que constituye el acto segundo del alma (In Met. I, Prol. n. 6 OPh, III, 5); y el tercero sostiene el deseo natural de asimilarse en grado sumo a las substancias separadas por medio de la contemplación de la verdad, acto perfecto y último de la intelección humana (In Met. I, Prol. n. 7 OPh, III, 5). Este último argumento se acompaña de una referencia al libro X de la Ética a Nicomaco (1177b2631), lo que permite a Escoto acentuar, a diferencia de Tomás, el medio que permite alcanzar esta asimilación a las sustancias separadas: la contemplación de la verdad.

Así pues, con el fin de reducir los tres argumentos a un solo, Escoto hace tres observaciones de las cuales consideraremos aquí la primera y la tercera:

(a) Apoyándose en la distinción expuesta por Aristóteles en el tratado Sobre el alma (II, 412a10-11), entre el acto primero y el acto segundo del alma, Escoto ve derivarse de allí la distinción de dos perfecciones: la de la forma y la de la operación. Esta es la razón por la cual construye una nueva premisa mayor que conduce en un solo movimiento a las conclusiones de los dos primeros argumentos de Tomás: «cada cosa desea naturalmente una y otra perfección, puesto que la operación es el fin del hábito». El segundo argumento de Tomás es pues reductible al primero gracias a una consideración de conjunto de los dos actos del alma (In Met. I, Prol. n. 8, OPh, III, 5-6)

(b) En segundo lugar, Escoto tampoco ve una diferencia entre el segundo y el tercer argumento, «puesto que le hombre únicamente es apto por naturaleza a unirse a su principio por medio de su operación. Y que no parece haber otra razón para desear la operación y tal unión» (In Met., I, Prol. n. 10, OPh, III, 6). Al final sólo queda un argumento sólido y compacto:

Cada uno naturalmente desea su perfección, tanto la primera, que es la forma, como la segunda, que es su operación propia, por la cual se une a su principio. Por lo tanto, el hombre naturalmente desea la ciencia, que es su perfección primera, y entender, que es su perfección segunda, por la cual se une a su principio (In Met. I, Prol. n. 11, OPh, III, 6-7).

Con este argumento o con sus versiones tomasianas, la relación entre lo inteligible en grado sumo y el sujeto de la metafísica se aclara. Es en razón del fin del hombre, determinado por el objeto de su deseo, el deseo de saber, que la metafísica se determina por lo inteligible en grado sumo. La consumación de la tarea metafísica es a un tiempo la consumación del fin del hombre y la actualización de su felicidad, la sabiduría.

\section{LAS DOS DIMENSIONES DE LA METAFÍSICA EN EL PRÓLOGO ESCOTISTA}

Después de este ajuste a la segunda prueba tomasiana de la dignidad y nobleza de la metafísica, Escoto se pregunta cuál es el objeto de esta ciencia:

Si todos los hombres desean por naturaleza saber, por lo tanto, desearán en grado sumo la ciencia en grado sumo. Así argumenta el Filósofo en el libro I capítulo 2 (982a30-b3). Y en el mismo lugar dice «que es ciencia en grado sumo (maxime scientia) 
aquella que se ocupa de las realidades cognoscibles en grado sumo (maxime scibilia)». Las realidades cognoscibles en grado sumo se entienden de dos maneras: $<\mathrm{A}\rangle$ porque son conocidas antes que todas las otras realidades (primo omnium) y sin ellas las otras realidades no pueden conocerse; $<\mathrm{B}>$ o porque son cognoscibles con máxima certeza (certissima cognoscibilia). Esta ciencia considera las realidades cognoscibles en grado sumo de los dos modos. Por lo tanto, esta es la ciencia en grado sumo y, en consecuencia, la ciencia deseable en grado sumo (maxime desiderabilis). (In Met. I, Prol. n. 16, OPh, III, 7-8).

Aquí vemos cómo se constituye el dominio metafísico en la determinación del concepto de maxime scibilia, las realidades cognoscibles en grado sumo, que pueden entenderse de dos maneras. Según la primera: (a) las maxime scibilia son las realidades «más comunes»o «comunísimas» (communissima) que Escoto identifica con el ente y sus propiedades trascendentales («ens in quantum ens, et quaecumque consequuntur ens in quantum ens» In Met. I, Prol. n. 17, OPh, III, 8). Este primer modo de las maxime scibilia «pertenece a la consideración de la metafísica (communissima pertinent ad considerationem metaphysicae)». Invocando el comienzo del libro IV $(\Gamma)$ de la Metafísica (1003a20-25), Escoto presenta una de las dos dimensiones constitutivas de este saber fundamental y la asocia al nombre «metafísica» que implica también la universalidad de esta ciencia:

Es necesario que haya una ciencia universal que considere por sí aquellas realidades trascendentes (quae per se consideret illa transcendentia). Y llamamos a esta ciencia «metafísica», cuyo nombre se construye a partir de «meta» que significa «trans» y de «ycos» que significa «ciencia», como una ciencia trascendental, porque es una ciencia que se ocupa de las realidades transcendentes. (Et hanc scientiam vocamus metaphysicam, quae dicitur a «meta», quod est «trans», et «ycos» «scientia», quasi transcendens scientia, quia est de transcendentibus). (In Met. I, Prol. n. 18, OPh, III, 9).

A propósito de este pasaje fundamental, que ha hecho correr mucha tinta entre los historiadores del escotismo, son necesarias varias observaciones. En primer lugar, con respecto a Tomás, notemos que Escoto procede también a dar un nombre, «metafísica», a esta dimensión universal de la ciencia buscada. En otras palabras, llamamos metafísica a la ciencia que se ocupa del ente y sus propiedades; y es con respecto a estas realidades que la consideramos como una scientia transcendens. En efecto, en su prólogo, siguiendo el enfoque de la universalidad en grado sumo, Tomás llamaba «metafísica» a la ciencia que se ocupa del ente y las propiedades que se derivan de él, y los llamaba «transphysica». No hay que olvidar que para Tomás, este enfoque se funda sobre la comparación del intelecto y los sentidos y por lo tanto, que su punto de partida es el contraste entre el conocimiento sensible que se ocupa del singular y el conocimiento intelectual que se ocupa del universal.

Por el contrario, gracias al recurso a la etimología de las partes de la palabra «metaphysica» formada por «trans» e «ycos», Escoto se separa de la interpretación tomasiana que insiste sobre la superación de la física, para llamar la atención sobre una superación de los objetos de todas las ciencias particulares gracias a la aprehensión inmediata de los transcendentia según un célebre argumento de Avicena, que Escoto hace suyo. ${ }^{36}$ De hecho, en tanto se

36 Avicenna, Philosophia prima sive scientia divina, I, 5 (AvL, I, 31-33): «Dicemus igitur quod res et ens et necesse talia sunt quod statim imprimuntur in anima prima impressione, quae non acquiritur ex aliis notioribus se, [...] Quae autem promptiora ad imaginandum per seipsa, sunt ea quae communia sunt omnibus rebus, sicut res et ens et unum, et cetera. Et ideo nullo modo potest manifestari aliquod horum probatione quae non sit circulari, vel per aliquid quod sit notius illis. Unde quisquis voluerit discurrere de illis incidet in involucrum, sicut ille qui dixit quod certitudo entis est quod vel est agens vel patiens : quamvis haec divisio sit entis, sed tamen ens notius 
ocupa del ser, esta ciencia es llamada metafísica tanto por Escoto como por Tomás, pero no por las mismas razones.

En efecto, consideramos que no se puede interpretar este pasaje sin tener en cuenta el argumento precedente que sostiene la necesidad de esta ciencia universal en tanto que ella se ocupa de aquello cuyo conocimiento previo es una condición necesaria para el conocimiento de los objetos de las otras ciencias (alia specialiora non possunt cognosci nisi illa communia prius cognoscantur) (In Met. I, Prol.n. 16, OPh, III, 7-8). Por el porcedimiento escotista, no se llega al ser por la via del análisis (via resolutionis) de Tomás, que toma por punto de partida lo menos común para dirigirse hacia lo más común, ${ }^{37}$ sino, más bien, por la vía aviceniana de las nociones comunísimas impresas inmediatamente en el alma.

Con respecto a este punto, L. Honnefelder, en sus conferencias parisinas sobre La métaphysique comme science transcendantale, ${ }^{38}$ concluye su presentación del modelo escotista de la metafísica como ciencia del ser unívioco, citando como confirmación final, esta pasaje del prólogo en el que la ciencia trascendental (scientia transcendens) o metafísica se ocupa de las transcendentia, comprendidas no como realidades separadas de la materia, sino como realidades transcategoríales. ${ }^{39}$ Sin embargo, incluso si ese es el caso de la concepción futura de la metafísica escotista, expuesta en su Ordinatio (I, d. 3 qq. 1-3, Vat. III, 1-123), en el hic et nunc de este prólogo, solo se trata de la caracterización de una de las dos dimensiones posibles de esta ciencia aún sin nombre. J. Aertsen muestra claramente, a propósito de este pasaje que comentamos ( $\$ 18)$, que no estamos frente a una innovación ni frente a una frase programática (scientia transcendens) en lo que concierne a la futura posición escotista sobre el dominio trascendental; por el contrario; el prólogo se situa en la línea de la larga tradición metafísica del siglo XIII. Habrá que esperar la Ordinatio con el fin de ver esta concepción de lo transcendental en acción. ${ }^{40}$ O. Boulnois le concede a Aertsen el carácter tradicional del prólogo, pero subraya el

est quam agens vel patiens. Omnes enim homines imaginant certitudinem entis, vel ignorant si debeat esse agens vel patiens; et mihi quousque nunc non patuit hoc nisi argumentatione tantum».

37 Thomas d'Aquin, In XII Met., Prooemium (ed. Cathala, p. 2) : «Metaphysica <dicitur>, inquantum considerat ens et ea quae consequuntur ipsum. Haec enim transphysica inveniuntur in via resolutionis, sicut magis communia post minus communia».

38 Honnefelder, L., La métaphysique comme science transcendantale: entre le Moyen Âge et les Temps modernes, trad. I. Mandrella, revisada por O. Boulnois, J. Greisch y Ph. Capelle, París, Presses Universitaires de France, 2002.

39 Ibid. «Deuxième leçon: La question de la portée de la raison humaine: la métaphysique comme science transcendantale», pp. 19-37: «Nous avons ainsi découvert la caractéristique décisive de la métaphysique scotiste: elle est la philosophie première recherchée en tant qu'elle est une "science transcendantale" (scientia transcendens). Et elle se présente ainsi, parce qu'elle est une science qui traite des "concepts transcendantaux" (transcendentia), à savoir du concept d" "étant" et des autres concepts qui participent au caractère transcatégorial du conceptus entis. La caractéristique décisive de la métaphysique n'est pas qu'elle traite des objets libres de matière et de changement, mais qu'elle traite des concepts transcatégoriaux. Ses objets ne sont ni l'ousia ni l'étant divin, mais le concept d'“étant"; et c'est seulement dans la mesure où l'ousia et l'étant divin sont appréhendés dans l'horizon de ce concept que la connaissance du premier Étant éminent est leur but». Aquí, pp. 36-37.

40 Aertsen, J., «Scotus's Conception of Transcendentality: Tradition and Innovation», en L. Honnefelder, H. Möhle, et alii. (eds.), Johannes Duns Scotus 1308-2008. Die philosophischen Perspektiven seines Werkes/Investigations into his Philosophy. «Archa Verbi: Yearbook for the Study of Medieval Theology - Subsidia 5», Münster-New York, Aschendorff Verlag-Franciscan Institute Publications, 2010, pp. 107-123, y para la crítica de la lectura de Honnefelder, pp. 108-112. Sobre el pasaje en cuenstión, Aertsen afirma: «The terminology is remarkable, but does not have a programmatic or polemical sense. On the contrary, Scotus's argument adopts the common understanding of transcendentals as the communissima in the thirteenth century...», pp. 108-109. Cf. Del mismo autor, Medieval Philosophy as Transcendental Thought: From Philip the Cancellor (ca. 1225) to Francisco Suárez, Leyde, Brill, 2012, pp. 372-375. 
hecho de que desde este primer período, Escoto se pregunta por la posibilidad de la metafísica como ciencia común. ${ }^{41}$

Así pues, si nos restringiéramos a esta primera dimensión que Escoto llama «metafísica», ya estaría plenamente presente en el prólogo la dimensión universal y trascendental de la ciencia primera por la que Escoto se célebre. Sin embargo, junto a ella, Escoto presenta una segunda dimensión que contrasta con la primera y excluye ver en el prólogo una primera afirmación de la metafísica como ontología.

Exploremos pues la segunda acepción de los maxime scibilia, las realidades cognoscibles con máxima certeza (certissimma cognoscibilia):

Las realidades cognoscibles con máxima certeza (certissima cognoscibilia) son principios y causas, y tanto más ciertas por sí cuanto más anteriores. De ellas depende toda certeza posterior. Esta ciencia considera de este modo los principios y las causas, como lo prueba el Filósoso en la Metafísica I, capítulo 2, en tanto llama a esta ciencia «sabiduría» (sapientia), como es manifiesto en el texto (In Met. I, Prol. n. 21, OPh, III, 10).

En esta segunda acepción de los maxime scibilia, la ciencia buscada es llamada «sabiduría» y se ocupa de los principios y causas primeros. Con esta segunda acepción recordamos de nuevo a Tomás y su primer enfoque sobre los maxime intelligibilia, a partir del orden de intelección.

Estas son pues las dos dimensiones de las maxime scibilia en el prólogo escotista: (a) en tanto communissima, corresponden al ente y sus propiedades; y (b) en tanto certissima cognoscibilia, corresponden a los primeros principios y a las primeras causas.

Si de acuerdo a la primera dimensión, nos situamos en un orden derivado de los grados de universalidad, puesto que los communisima son la condición necesaria del conocimiento de las otras realidades; de acuerdo a la segunda dimensión, nos situamos en un orden derivado de ciertos grados de potencia causal expresados por grados de certeza. En ese sentido, el concepto de certeza expresa el grado de constitución ontológica de los maxime scibilia, antes que una propiedad epistémica o noética. Sin embargo, al menos hasta ese momento, Escoto no los identifica con Dios y las inteligencias.

En el esquema que se deriva de esta doble dimensión, Escoto reduce la tripartición tomasiana a una bipartición en la cual, por ahora, no vemos un lugar o un rol determinado para Dios y las inteligencias. A esta situación podemos además agregar, la reorganización de los nombres y la ausencia de una función explícitamente reguladora para esta ciencia; función que estructuraba toda la reflexión del prólogo tomasiano alrededor de la idea de una regla del intelecto. En una palabra, en el prólogo escotista es manifiesta la ausencia de una estructura explícita de la metafísica como ciencia. Por el contrario, el prólogo acentúa de nuevo la tensión metafísica en la determinación de su objeto que se mueve entre la opción por el ente y la opción por los primeros principios y las primeras causas.

41 Boulnois, O., Métaphysiques rebelles, op. cit., p. 263 y n. 3: «Il y a donc dans la première rédaction de ses questions sur la Métaphysique, une ambivalence non résorbée entre deux sujets et deux dimensions de la métaphysique: la considération des transcendantaux, et l'enquête sur les premières causes. [...] Mais on ne peut nier que dès cette époque, Duns Scot met tout son effort sur l'analyse de la possibilité d'une science transcendantale». 


\section{OBSERVACIONES FINALES}

Si el prólogo de Escoto está menos estructurado que el de Tomás, es en razón de una indeterminación que nosotros creemos consciente a propósito del sujeto propio de esta ciencia. Como lo acabamos de mencionar, en este texto aún no encontramos al célebre Escoto de la univocidad del ser y de la metafísica como ciencia del ser. A este respecto, Tomás establece claramente la relación entre el ens commune sujeto de la sapientia y las substancias separadas que juegan precisamente el rol de causas primeras del objeto. En cuanto a Escoto, su vacilación anuncia el gran debate que tendrá lugar en la primera cuestión del primer libro del comentario, donde, como ya lo mencionamos, se ocupa precisamente del problema de la determinación del objeto de la metafísica. Sin embargo, en ese lugar la respuesta no solamente va a mantenerse indeterminada, o al menos confusa, sino que allí Escoto propondrá y defenderá, junto a los candidatos tradicionales (el ente o Dios y las inteligencias) asociados a los nombres de Avicena y Averroes, un nuevo candidato: la substancia (In Met., I, q. $1 \mathrm{nn}$. 91-109, OPh, III, 46-53).

El prólogo escotista a las Cuestiones sobre la Metafísica, en su indeterminación, vacila entre el ente y las primeras causas como objeto de esta ciencia, mientras Tomás hace del ente tanto el objeto de la metafísica como el primer efecto de las primeras causas. Es verdad que con la caracterización de la metafísica como scientia transcendens se anuncia la solución escotista de la univocidad del ente, pero en este prólogo, ella es una alternativa. En ese sentido, el prólogo de Escoto se aleja de las certezas del prólogo tomasiano, a pesar de los ajustes que haya podido hacerle, $\mathrm{y}$, antes de defender la univocidad, reabre la pregunta por el sujeto y la estructura de la metafísica.

\section{BIBLIOGRAFÍA}

\section{Fuentes primarias}

Aristoteles Latinus, Metaphysica, lib. I-X, XII-XIV. Translatio Anonyma sive 'Media', ed. G. Vuillemin-Diem, «Aristoteles Latinus, XXV 2», Leiden, E.J. Brill, 1976.

Avicenna, Liber de philosophia prima sive Scientia divina I-IV, ed. S. Van Riet; introducción doctrinal G. Verbeke, «Avicenna Latinus», Lovaina-Leiden, Peeters-Brill, 1977.

Dominicus Gundissalinus, De divisione philosophiae, ed. L. Baur, «Beiträge zur Geschichte der Philosophie des Mittelalters: Texte und Untersuchungen, Band 4, Heft 2-3», Münster, Aschendorff, 1903.

Juan Duns Escoto, Opera omnia III: Ordinatio I, d. 3, ed. C. Balić et al., Civitas Vaticana, Typis Polyglottis, 1954.

- Opera philosophica III-IV: Quaestiones super libros metaphysicorum Aristotelis, ed. R. Andrews et al., St. Bonaventure (NY), The Franciscan Institute, 1997.

John Duns Scotus, Questions on the Metaphysics of Aristotle, II Vols., translated by Girard J. Etzkorn \& Allan B. Wolter, O.F.M., «Franciscan Institute Publications, Texts Series 19 », St. Bonaventure (NY), Franciscan Institute Publications, 1997-1998.

Tomás de Aquino, In Duodecim Libros Metaphysicorum Aristotelis expositio, eds. M. R. Cathala y R. M. Spiazzi, Turín-Roma, Marietti, 1950.

\section{Bibliografía secundaria}

Aertsen, J., «Scotus's Conception of Transcendentality: Tradition and Innovation», en L. Honnefelder, H. Möhle, et alii. (eds.), Johannes Duns Scotus 1308-2008. Die philosophischen 
Perspektiven seines Werkes/ Investigations into his Philosophy. Proceedings of «The Quadruple Congress» on John Duns Scotus, Part 3, «Archa Verbi: Yearbook for the Study of Medieval Theology - Subsidia 5», Münster-New York: Aschendorff Verlag-Franciscan Institute Publications, 2010, pp. 107-123.

Aertsen, J., Medieval Philosophy as Transcendental Thought: From Philip the Cancellor (ca. 1225) to Francisco Suárez, «Studien und Texte zur Geistesgeschichte des Mittelalters, 107», Leyde, Brill, 2012.

Aubenque, P., Le problème de l'être chez Aristote. Essai sur la problématique aristotélicienne, «Épiméthée», París, Presses Universitaires de France, 1962.

Boulnois, O., «Analogie et univocité selon Duns Scot: La double destruction», Les Études philosophiques, «L'analogie», (1989) 3-4, pp. 347-369.

- «La métaphysique selon saint Thomas d'Aquin. Lecture du «Prologue» de son commentaire d'Aristote», en T.-D. Humbrecht (dir.), Saint Thomas d'Aquin, «Les Cahiers d'Histoire de la Philosophie», París, Les Éditions du Cerf, 2010, pp. 37-88.

- Métaphysiques rebelles: Genèse et structures d'une science au Moyen Âge, «Épiméthée», París, Presses Universitaires de France, 2013.

Courtine, J.-F., Suarez et le système de la métaphysique, «Épiméthée», París, Presses Universitaires de France, 1990.

Cross, R., Duns Scotus's Theory of Cognition, Oxford, Oxford University Press, 2014.

Demange, D., «L'objet de la philosophie première selon Thomas d'Aquin et Jean Duns Scot», Le Philosophoire, 20 (2003), pp. 173-196.

Dod, B., «Aristoteles Latinus», en Kretzmann N., Kenny A. et Pinborg J. (eds.). The Cambridge History of Later Medieval Philosophy. From the Rediscovery of Aristotle to the Disintegration of Scholasticism, 1100-1600, Cambridge, Cambridge Univesity Press, 1982, pp. 45-79.

Doig, J. C., Aquinas on Metaphysics: A Historico-doctrinal Study of the Commentary on the Metaphysics, La Haye, Martinus Nijhoff, 1972.

Gilson, É., Jean Duns Scot: Introduction à ses positions fondamentales, «Études de philosophie médiévale, xli », París, Vrin, 1952.

Honnefelder, L., La métaphysique comme science transcendantale: entre le Moyen Âge et les Temps modernes, trad. I. Mandrella, revisada por O. Boulnois, J. Greisch y Ph. Capelle, «Chaire Étienne Gilson», París, Presses Universitaires de France, 2002.

Libera, A. de., «Les sources gréco-arabes de la théorie médiévale de l'analogie de l'être», Les Études philosophiques, «L'analogie», 3-4 (1989), pp. 321-345.

- La querelle des Universaux. De Platon à la fin du Moyen Âge, París, Seuil, 1996.

- La philosophie médiévale, París, Presses Universitaires de France, 1998.

Marenbon, J., «The Latin Tradition of Logic to 1100», D. M. Gabbay y J. Woods (eds.), Handbook of the History of Logic. Vol. 2: Mediaeval and Renaissance Logic, Amsterdam, North-Holland, 2008, pp. 1-63.

Ramón Guerrero, R., «Avicena y Averroes en las Quaestiones subtilissimae super libros metaphysicorum Aristotelis de Duns Escoto», XII Congreso Latinoamericano de Filosofía Medieval. Juan Duns Scoto, Buenos Aires, FEPAI, 2008.

Torrell, J.-P., Saint Thomas d'Aquin: L'homme et l'œuvre, París, Les éditions du Cerf, 2012.

Trizio, M., Gutas, D. \& Burnett, Ch., «Apendix B: Medieval translations 1-3». R. Pasnau y Ch. van Dyke (eds.), The Cambridge History of Medieval Philosophy, vol. II, Cambridge, Cambridge University Press, 2010, pp. 793-822. 
Wippel, J. F., «Thomas Aquinas's Commentary on Aristotle's Metaphysics», en J. F. Wippel, Metaphysical Themes in Thomas Aquinas II, «Studies in Philosophy and the History of Philosophy, 47», Washington, The Catholic University of America Press, 2007, pp. 240271.

- «The Latin Avicenna as a Source for Thomas Aquinas's Metaphysics», en J. F. Wippel, Metaphysical Themes in Thomas Aquinas II, «Studies in Philosophy and the History of Philosophy, 47», Washington, The Catholic University of America Press, 2007, pp. 31-64.

Wolter, A. B., «Reflections on the Life and Works of Scotus», American Catholic Philosophical Quarterly, 67/1 (1993), pp. 1-36.

— «Reflections about Scotus's Early Works», en L. Honnefelder, R. Wood y Mechthild Dreyer (eds.), John Duns Scot: Metaphysics and Ethics, «Studien und Texte zur Geistesgeschichte des Mittelalters, 53», Leyde, Brill, 1996, pp. 37-57.

Wood, R., «The Subject of the Aristotelian Science of Metaphysics», en R. Pasnau y Ch. van Dyke (eds.), The Cambridge History of Medieval Philosophy, vol. II, Cambridge, Cambridge University Press, 2010, pp. 609-621.

Zimmermann, A., Ontologie oder Metaphysik? Die Diskussion über den Gegenstand der Metaphysik im 13. Und 14. Jahrhundert. Texte und Untersuchungen, «Studien und Texte zur Geistesgeschichte des Mittelalters, 8», Leyden-Colonia, Brill, 1965.

Fecha de recepción: día 1 de julio de 2016

hsalinas@javeriana.edu.co

Fecha de aceptación: día 9 de septiembre de 2016 